\title{
Using mHealth to Promote Mothers' Brushing of Preschool Children's Teeth: A Study Protocol using the Multi-phase Optimization Strategy (MOST)
}

Merna Ihab ( $\nabla$ merna.ihab@alexu.edu.eg )

Alexandria University Faculty of Dentistry https://orcid.org/0000-0002-7281-7381

Wafaa Essam El Din

Alexandria University Faculty of Dentistry

Nour Ammar

Alexandria University Faculty of Dentistry

Randa Yassin

Alexandria University Faculty of Dentistry

Maha El Tantawi

Alexandria University Faculty of Dentistry

\section{Research Article}

Keywords: Multi-phase optimization strategy, MOST, optimization trial, preparation phase, oral hygiene, motivational interviewing, mHealth, preschoolers, early childhood caries

Posted Date: October 29th, 2021

DOI: https://doi.org/10.21203/rs.3.rs-733148/v2

License: (c) (1) This work is licensed under a Creative Commons Attribution 4.0 International License. Read Full License 


\section{Abstract}

\section{Background}

Early childhood caries is a highly prevalent disease affecting young children. Parental brushing of children's teeth is recommended during preschool years. Interventions to promote parental brushing of children's teeth are assessed as a package in randomized clinical trials and the efficacy of separate components is not known.

\section{Methods and Analysis}

The aim of this study is to develop an optimized behavior modification intervention to increase mothers' brushing of their pre-school children's teeth using the Multi-phase Optimization Strategy (MOST) guided by the Theory of Planned Behavior. Behavior change will be assessed by the percent reduction in children's dental plaque index after 6 months and mothers' reporting of toothbrushing frequency. Two phases of MOST will be carried out. First: the preparation phase comprises the development of a conceptual framework, identifying candidate components, conducting a feasibility pilot study to assess the acceptability and the design features of three intervention components (motivational interviewing (Ml), and two mobile health (mHealth) components: oral health promotion messages, and storytelling videos delivered using WhatsApp messenger) in addition to setting an optimization objective. Second: the optimization phase constitutes a factorial trial assessing the three intervention components and developing the intervention by selecting the most effective components within the optimization constraint. Each component will be set at two levels: yes (the intervention is applied) and no (the intervention is not applied). A linear regression model will be used to assess the effect of the intervention components on the percent reduction in dental plaque index (primary outcome measure). The secondary outcome measure is the change in the frequency of mother's brushing of the child's teeth. The combination of components making up the new optimized intervention will be selected.

\section{Discussion}

This will be the first study to apply the MOST framework in the field of dentistry. The results of this study can guide to the development of an optimized behaviour modification interventions using mHealth and MI.

\section{Trial Registration}

This trial was registered on June $11^{\text {th }}, 2021$, in Clinical trials.gov. Registration number: NCT04923581.

\section{Background}

Early childhood caries (ECC) is the most prevalent chronic disease affecting children worldwide with high prevalence in Egypt exceeding 50\% [1, 2]. ECC has adverse effects on the growth and development of children and their quality of life as well as that of their caregivers [3]. Regular toothbrushing with 
fluoridated toothpaste is a highly effective home care measure [4], which renders dental health education of caregivers one of the best approaches to control this disease [5].

Parents are responsible for brushing their children's teeth at young age to prevent dental caries [6, 7]. The American Academy of Pediatric Dentistry recommends parental supervision of children's toothbrushing during preschool years [8]. Barriers to mothers' brushing of their children's teeth include inadequately supportive and organized home environment, absence of flexibility in daily activities, and parenting styles lacking positive reinforcement and involvement $[9,10]$. In addition, the child's development, level of cooperation, and the surrounding community-level factors may influence toothbrushing [10].

Several theories were proposed to explain health behaviors and how they are affected by various factors and barriers. These theories include the social cognitive theory, the theory of diffusion of innovation, the theory of planned behaviour (TPB) [11], and the health belief model [12]. The TBP was used to decipher barriers to behaviour change in oral health education programs [13], to design behaviour change interventions [14], to improve interventions by assessing participants' background [15], and to promote parental brushing of children's teeth [16]. The TPB can guide the development of interventions to promote mothers' brushing of their children's teeth by addressing the impact of barriers affecting mothers' perceived control, their attitude toward oral health, and the norms they perceive regarding the value of oral hygiene.

Several interventions were developed [17] to promote parents' brushing of children's teeth and were assessed using randomized clinical trials (RCTs). These interventions usually consist of multiple components to maximize the efficacy of the intervention and are evaluated as a single package [18]. The multi-phase optimization strategy (MOST) assesses the effectiveness of each component in the intervention separately. MOST is an engineering-inspired framework that focuses on developing and optimizing an intervention according to a specific optimization objective so that components of the interventions can later be evaluated for effectiveness as a package in an RCT [18]. The aim is not only to develop an effective intervention but an efficient one too. Understanding the mechanisms by which an intervention produces its effects makes it possible to build upon previous interventions in a more systematic and gradual means [18]. MOST consists of three phases: preparation, optimization, and evaluation phases. The preparation phase lays the groundwork for the development of the conceptual framework and the identification of candidate intervention components using a feasibility pilot study. In the optimization phase, the optimized intervention is constructed using optimization trials. Finally, the effectiveness of the optimized intervention can be confirmed in the evaluation phase through an RCT.

We aim to use the MOST framework to engineer a behavior modification intervention to promote mothers' brushing of their pre-school children's teeth using fluoridated toothpaste and to select intervention components and component levels with the greatest efficacy within a predefined optimization objective. We will select intervention components based on the constructs of the TPB then test them for acceptability in a feasibility pilot study representing the preparation phase of MOST. This will be followed 
by an optimization factorial design trial to compare the selected components and select the components with the greatest efficacy within predefined optimization objectives.

\section{Methods/design}

\section{Overview of the study}

I- The preparation phase, including:

1. Developing a conceptual framework to guide the design of the intervention.

2. Identifying candidate intervention components.

3. Conducting a feasibility pilot study to determine the acceptability of the intervention components and identify the preferred frequency and timing of providing them.

4. Setting an optimization objective.

II- The optimization phase, including:

1. Assessing the individual components using a factorial trial.

2. Identifying the intervention components with greatest efficacy with the specified optimization objective.

\section{Preparation Phase}

\section{a. Conceptual Framework}

The TPB posits that intentions are predictors of behaviours and are affected by attitudes, subjective norms, and perceived control [19]. Individuals adopt beliefs about the outcomes of a behavior, and these beliefs contribute to their attitude. Subjective norms are the perception about the expectations of important others, and these contribute to the perception of social pressure and motivation to comply. Perceived control is divided into two components self- efficacy which is the sense of ease/ difficulty in engaging in the behavior; and controllability which is the extent to which the performance is up to the person. Parental attitude, self-efficacy, and intention are modifiable social-cognitive constructs that were reported to be significantly associated with preschool children's oral hygiene behavior and can be targeted by behaviour modification interventions [20]. In the proposed study, each TPB construct will be targeted by a component, and this is assumed to lead to the desired behaviour modification (Figure 1).

\section{b. Identifying candidate components (Table 1)}

Three components are proposed in addition to a core/constant component provided to all participants. The three components are: motivational interviewing (MI), oral health promotion messages (OHPM), and storytelling (ST). In a previous study, MI outperformed traditional oral health education in improving patient behaviors and oral health perceptions and enhancing clinical indicators (e.g., plaque index, 
gingival index, bleeding on probing) [21]. Another study [22] reported that messages based on subjective norms were the most effective in inducing behavioural changes. The use of story-based approaches also showed promising results in improving children's oral hygiene [23].

\section{Constant component}

All participants will receive pamphlets emphasizing the importance of primary teeth, correct feeding habits, recommended sugar intake, and age-appropriate oral hygiene practices. Toothpaste samples will be distributed as tokens of appreciation. This component aims to provide standard basic oral health education and foster engagement and trust.

\section{Motivational Interviewing}

A single 30-45-minute MI session will be offered. This component will target the perceived control construct of the TPB. The researcher providing the MI will receive training using the MI network of trainers' resources [24]. The MI session includes establishing rapport and encouraging participants to talk about their dental habits and what they want for their children to motivate them to accept changes in behaviors. Options and strategies for improving dental health will be discussed. After the session, parents will be contacted by phone every two months, for 6 months, to reinforce commitment to the new behaviour, and provide support. Without follow-up, new behaviours may not be maintained or even tried leading to relapse.

\section{Oral Health Promotion Messages}

Teledentistry and mobile health (mHealth) are proving to be an effective tool for the promotion of oral health in various age groups [25]. mHealth components will be incorporated in the proposed study to reduce the time needed for mothers to receive health education, adhere to physical distancing in the time of COVID-19 and make use of the popularity of these tools among the younger generations. A series of unique messages targeting the subjective norms construct of the TBP will be sent to the mothers using the WhatsApp Messenger [26]. The messages are based on previous studies [23,27] and are modified to fit the proposed study. The validity, acceptability, preferred frequency, and timing of messages will be determined in the feasibility pilot study of the preparation phase (Appendix 1). Based on the frequency, the number of messages will be determined so that they are spread over the study period.

The following are examples of the messages:

"\{name\}, this is \{dentist\} from \{clinic\}. Did you know that brushing your children's teeth twice daily using a toothbrush and toothpaste will help them have a nicer smile in front of their friends and family?"

"\{name\}, this is \{dentist\} from \{clinic\}. Ensure smaller dental bills for your family by brushing your child's teeth twice daily using a toothbrush and toothpaste." 
"\{name\}, this is \{dentist\} from \{clinic\}. Be a role model for your children and show them how to brush their teeth twice daily using a toothbrush and toothpaste".

\section{Storytelling Videos}

Via WhatsApp messenger, the mothers will receive another mHealth component: a series of 1-minute videos targeting the attitude construct of the TPB. The videos have a scenario narrating the experiences of mothers whose children suffered from ECC, emphasizing the positive effect of tooth brushing and fluoridated toothpaste in controlling ECC, and enhancing children's oral hygiene. The preferred frequency and timing of the videos will also be assessed during the preparation phase (Appendix 1) and based on the frequency; the number of videos will be determined.

\section{Table 1. Candidate Intervention Components}

\begin{tabular}{|llll|}
\hline $\begin{array}{l}\text { Intervention } \\
\text { component }\end{array}$ & Target construct & $\begin{array}{l}\text { Frequency of delivering } \\
\text { the component }\end{array}$ & Method of Delivery and setting \\
\hline $\begin{array}{l}\text { Constant } \\
\text { component }\end{array}$ & Core knowledge & Once at baseline & Pamphlets in clinic \\
\hline MI & $\begin{array}{l}\text { Perception of } \\
\text { control }\end{array}$ & $\begin{array}{l}1 \mathrm{Ml} \text { session }+3 \text { phone } \\
\text { calls }\end{array}$ & $\begin{array}{l}\text { Face to face in clinic + phone } \\
\text { calls }\end{array}$ \\
\hline OHPM & $\begin{array}{l}\text { Perceived } \\
\text { norms }\end{array}$ & $\begin{array}{l}\text { Determined during the } \\
\text { feasibility pilot study }\end{array}$ & $\begin{array}{l}\text { mHealth: electronic messages via } \\
\text { WhatsApp messenger }\end{array}$ \\
\hline ST videos & Attitude & $\begin{array}{l}\text { Determined during the } \\
\text { feasibility pilot study }\end{array}$ & $\begin{array}{l}\text { mHealth: electronic messages via } \\
\text { WhatsApp messenger }\end{array}$ \\
\hline
\end{tabular}

\section{c. Feasibility Pilot Study}

The feasibility pilot study assesses the acceptability and features of MI, OHPM, and ST videos. The feasibility study is not planned to be powered. Ten to 16 mothers will be recruited from the clinic of the Department of Pediatric Dentistry and Dental Public Health, Faculty of Dentistry, Alexandria University, Alexandria, Egypt until information saturation occurs [28]. Each mother will receive and provide feedback on the MI session and phone calls, the OHPM and the ST videos.

\section{Inclusion criteria for mothers in the feasibility pilot study:}

1. Has children between 2 and $<5$ years of age.

2. Has children with dental plaque index scores $\geq 2$ or visible plaque accumulation on maxillary anterior teeth.

3. Literate and owns a mobile phone with WhatsApp messenger application or where WhatsApp messenger can be installed. 
4. Willing to participate in the study.

\section{Exclusion criteria:}

1. Mothers of children with a definitely negative behavior (Frankel's scale rating 4) [29].

2. Mothers of physically disabled or medically compromised children.

3. Mothers of children who need emergency dental treatment.

The mother will sign an informed consent form and receive the 3 components. The acceptability of the 3 components will be evaluated in a semi-structured interview using the framework of acceptability [30] assessing: affective attitude, burden, perceived effectiveness, ethicality, intervention coherence, and opportunity costs (Appendix 1). They will also be asked about the preferred frequency and timing of the messages and videos.

\section{d. Setting the optimization objective}

The optimization objective is to develop an intervention that fits within the time a mother is willing to spend receiving health education to support her to brush her child's teeth. This objective may be more relevant in the mother and child health centers where the optimized intervention is planned to be provided. In this setting, mothers receive ante and post-natal care including health education. These services are provided free of charge by salaried providers and thus, financial constraints and provider time may not be appropriate constraining factors. The key constraint [19] in the present study is the time the mother is willing to spend receiving the components. This time should cover commuting to and from the clinic to receive $\mathrm{MI}$, the time of the phone call reminders for $\mathrm{Ml}$, the time to read the WhatsApp messages and that spent watching the videos. It will be assessed using a questionnaire to the mothers in the feasibility pilot study (Appendix 1).

\section{II- Optimization Phase}

\section{a. Factorial trial design}

A factorial trial will be used to compare the levels of the three intervention components: MI, OHPM, and ST videos with the features identified during the preparation phase. Factorial designs are preferred for comparing the three components for two reasons. Firstly, factorial experiments separate component effects, enabling the estimation of the main effect and interactions between components. Secondly, factorial trials are efficient compared to alternative designs because they require fewer participants to achieve the same statistical power. Conducting three individual trials will require three times as many participants as the factorial trial.

\section{Study Setting}

This trial will be conducted at the outpatient clinic of the Department of Pediatric Dentistry and Dental Public Health, Faculty of Dentistry, Alexandria University. 


\section{Eligibility Criteria}

The inclusion and exclusion criteria are the same as those of the feasibility pilot study.

\section{Intervention components}

Each of the three components (MI, OHPM and ST) has two levels: yes (the intervention is applied) and no (the intervention is not applied) as shown in Table 2. Participants will be divided into subgroups, each receiving one of the eight experimental conditions.

Table 2. Factorial Trial Design in the Optimization Phase with 8 Experimental Conditions

\begin{tabular}{|llll|}
\hline Experimental Condition & MI & OHPM & ST \\
\hline $\mathbf{1}$ & No & No & No \\
\hline $\mathbf{2}$ & No & No & Yes \\
\hline $\mathbf{3}$ & No & Yes & No \\
\hline $\mathbf{4}$ & No & Yes & Yes \\
\hline $\mathbf{5}$ & Yes & No & No \\
\hline $\mathbf{6}$ & Yes & No & Yes \\
\hline $\mathbf{7}$ & Yes & Yes & No \\
\hline $\mathbf{8}$ & Yes & Yes & Yes \\
\hline
\end{tabular}

\section{Outcome Measures}

The outcome assessment uses validated methods and assessment tools including a clinical examination and a questionnaire. Therefore, these tools and methods will not be further assessed in the pilot feasibility study.

1. Primary outcome: Reduction in the dental plaque index scores of children of participating mothers after 3 and 6 months [31], measured using the modified plaque index (PII) of Silness and Löe [32]. The World Health Organization (WHO) community periodontal index of treatment needs (CPITN) probe and a disposable dental mirror will be used to assess plaque on 6 index teeth (upper right $2^{\text {nd }}$ primary molar, upper primary right lateral incisor, upper left $1^{\text {st }}$ primary molar, lower left $2^{\text {nd }}$ primary molar, lower left primary lateral incisor, and lower right $1^{\text {st }}$ primary molar) at baseline and after 3 and 6 months. The four surfaces of each tooth will be scored from 0 to 3 and the scores will be averaged per tooth. The scores of the six teeth will be averaged to obtain the mean PII of the child. The outcome measure is the percent 
reduction in dental plaque scores calculated as: [(plaque score after 3 or 6 months- plaque score at baseline)/ plaque score at baseline] *100.

2. Secondary outcome: Mother-reported frequency of brushing the child's teeth using fluoridated toothpaste will be assessed by the Arabic version of the WHO questionnaire [33] at baseline, after 3 and 6 months. The questionnaire assesses the frequency of toothbrushing on a 7-point scale ranging from never to twice or more a day. The outcome measure is the change in frequency from baseline to 3 or 6 months categorized as increased (changing from lower to higher frequency), remained the same (reporting the same frequency) and decreased (changing from higher to lower frequency). (Appendix 2)

3. Moderators such as child's age and sex, the number of children in the family, mothers' age, oral hygiene practices, and education level will be assessed by the questionnaire.

\section{Participant Timeline}

\section{Sample Size Calculation}

The study is powered to detect at least a medium effect size measured by partial eta squared $\left(\mathbb{\triangle}^{2}\right)=0.06$; the effect size measure for factorial ANOVA [34]. In $G *$ Power 3.0.10 [35], we specified $f=0.0 .2526456$ based on the required $\mathbb{\bigotimes}^{2}$, alpha error $=5 \%$ and study power $=80 \%$. For the 8 conditions in Table 2 , we used numerator $\mathrm{df}=(2$ levels in MI- 1$) \times(2$ levels in OHPM- 1$) \times(2$ levels in ST videos- 1$)=1 \times 1 \times 1=1$. A total sample size of 126 will allow the estimation of main effects and interactions using linear regression analysis. This will be rounded to 160 corresponding to 20 mothers in each of the 8 experimental conditions.

\section{Randomization and Allocation Concealment}

Mothers will be assessed for eligibility and randomly allocated to the 8 experimental conditions (Table 2). The Random Allocation Software [36] will be used to generate a randomization schedule which will be kept in opaque, sealed envelopes. The envelopes will be arranged sequentially by a dental assistant, who will not be involved in the study. Each envelope will be opened after completing the child's oral examination at baseline and the experimental condition to which the mother is allocated will be administered. Mothers' enrollment, random sequence generation and allocation to experimental conditions will be done by independent researchers who will be different from the researcher implementing the experimental conditions and assessing the outcomes.

\section{b. Selecting the most efficacious components fitting the optimization objective}

The combination of components making up the optimized intervention will be selected according to the following procedure [37]:

- The three components are labeled $A$ to $C$. 
- The main effect of component A will be defined as the difference between the yes level and the no level of that component, averaged across all the levels of components $B-C$. This will be calculated by subtracting the mean response in conditions 1-4 in Table 2 from the mean response in conditions 5-8.

- A two-way interaction involving components $A$ and $B$ will occur if the effect of $A$ at the "Yes" level of $B$ is different from the effect of $A$ at the "No" level of $B$, averaged across all the levels of $C$ and $D$.

- Regression coefficient estimates will be produced by conducting a factorial ANOVA using effect coding $(-1,1)$.

- If a component has a main effect with at least a moderate effect size, the "yes" level will be chosen for inclusion in the intervention. If the component does not achieve a moderate effect size or has an effect in the wrong direction, such as increasing the plaque index, the "no" level will be selected. This allows the inclusion of efficacious components and the elimination of components which have no use.

- An initially selected component may be deselected if it interacts with another component which undermines its effect, or a component not initially selected may be selected if it interacts with another component to enhance its effect.

- The combination of components which fits within the constraint of the optimization objective will be selected for inclusion in the optimized intervention package.

\section{Data Management and Statistical Analysis}

IBM SPSS Statistics for Windows (Version 25.0. Armonk, NY: IBM Corp) [38] will be used for data analysis. A linear regression model will be used to assess the effect of the components on percent reduction in plaque index score adjusting for confounders.

\section{Discussion}

This study adopts an innovative research method to develop an efficient and scalable behavior modification intervention based on participants' acceptance and preferences using components that are likely to accommodate the conditions during the time of the pandemic. The literature about young children's oral hygiene provides little guidance on optimal intervention designs and existing evidence describes components which vary in effectiveness. Little is known from the literature about the underlying theoretical framework, form, and dose of interventions to elicit effective behaviour change. Previous studies [39] used packages of multiple interventions but none of them assessed the efficacy of individual components. Our study is considered the first to apply the MOST framework in the field of dentistry. Through MOST, we hope to isolate the components then assemble an efficacious standalone intervention package that fits the optimization criterion.

\section{Trial Status}


This is the original version of the protocol, issued on the 9th of June 2021. The recruitment is planned to start on the 20th of August 2021 and end approximately by February 2022. Any changes or protocol amendments will be made to the study record available on clinical trials.gov.

\section{Abbreviations}

ECC: Early Childhood Caries

MOST: Multi-Phase Optimization Strategy

RCT's: Randomized Clinical Trials

TPB: Theory of Planned Behaviour

Ml: Motivational Interviewing

OHPM: Oral Health Promotion Messages

ST: Storytelling

\section{Declarations}

\section{Ethics Approval and Consent to Participate}

The study received approval from the Ethics Committee of the Faculty of Dentistry, Alexandria University. IRB No: 00010556-IORG: 0008839. A signed informed consent will be obtained from each mother after explaining the objectives, risks, and benefits of the study. The voluntary nature of the study will be emphasized in the consent form, and the mothers will also be informed of their right to withdraw from the trial anytime, without incurring any penalties.

\section{Confidentiality}

Identification numbers will be used to assure participant confidentiality during data analysis. All data will be collected using a secure, web-based, and password-protected database.

\section{Consent for Publication}

Not applicable.

\section{Availability of Data and Materials}

The final dataset of the proposed study will be available upon reasonable request from the corresponding author.

\section{Competing Interests}


The authors have no competing interests to declare.

\section{Funding}

This study did not receive any grant from funding agencies in the public, commercial, or not-for-profit sectors.

\section{Authors' Contributions}

MI is the study's principal investigator. MET and MI conceptualized, designed the study, and drafted the manuscript. WE, NA, and RY revised and reviewed the manuscript. All authors contributed to the final approval of the manuscript.

\section{Acknowledgements}

Not applicable.

\section{References}

1. Fadl AE, Abdel Fattah R, Ezz M. M. Assessing the prevalence of early childhood caries and the associated determinants in a group of preschool children: results from a national oral health survey in Egypt. Egypt Dent J. 2019;65:31-9.

2. El Tantawi M, Folayan MO, Mehaina M, Vukovic A, Castillo JL, Gaffar BO, et al. Prevalence and data availability of early childhood caries in 193 united nations countries, 2007-2017. Am J Public Health. 2018;108:1066-72.

3. Sheiham A. Dental caries affects body weight, growth and quality of life in pre-school children. $\mathrm{Br}$ Dent J. 2006;201(10):625-6.

4. Horst JA, Tanzer JM, Milgrom PM. Fluorides and Other Preventive Strategies for Tooth Decay. Dent Clin North Am. 2018;62(2):207-34.

5. Boustedt K, Dahlgren J, Twetman S, Roswall J. Tooth brushing habits and prevalence of early childhood caries: a prospective cohort study. Eur Arch Paediatr Dent. 2020;21:155-9.

6. Clarke R, Shaw-Ridley M. Parental Attitudes and Beliefs About Preschooler Preventive Oral Health Behaviors: Implications for Health Promotion. J Immigr Minor Heal. 2019;21:731-6.

7. Hamilton K, Cornish S, Kirkpatrick A, Kroon J, Schwarzer R. Parental supervision for their children's toothbrushing: Mediating effects of planning, self-efficacy, and action control. Br J Health Psychol. 2018;23:387-406.

8. American Academy of Pediatric Dentistry. Fast Facts.https://www.aapd.org/assets/1/7/FastFacts (accessed 10 July 2021).

9. Duijster D, de Jong-Lenters M, Verrips E, Loveren C van. Establishing oral health promoting behaviours in children - parents' views on barriers, facilitators and professional support: A qualitative study. BMC Oral Health. 2015;15:1-13. 
10. Finlayson TL, Cabudol M, Liu JX, Garza JR, Gansky SA, Ramos-Gomez F. A qualitative study of the multi-level influences on oral hygiene practices for young children in an Early Head Start program. BMC Oral Health. 2019;19:1-14.

11. Ajzen I. The theory of planned behavior. Organ Behav Hum Decis Process Academic Press. 1991;50:179-211.

12. Davis R, Campbell R, Hildon Z, Hobbs L, Michie S. Theories of behaviour and behaviour change across the social and behavioural sciences: a scoping review. Health Psychol Rev. 2015;9:323-44.

13. Amin M, Nyachhyon P, Elyasi M, Al-Nuaimi M. Impact of an Oral Health Education Workshop on Parents' Oral Health Knowledge, Attitude, and Perceived Behavioral Control among African Immigrants. J Oral Dis. Hindawi Limited. 2014; 2014:1-7.

14. Hardeman W, Johnston M, Johnston D, Bonetti D, Wareham N, Kinmonth AL. Application of the theory of planned behaviour in behaviour change interventions: A systematic review. Psychol Heal. 2002;17:123-58.

15. Mathieson K. Predicting user intentions: Comparing the technology acceptance model with the theory of planned behavior. Inf Syst Res. 1991;2:173-91.

16. Wang K, Hiu G, Lee M, Liu P, Gao X, Yeung S, et al. Health Belief Model for Empowering Parental Toothbrushing and Sugar Intake Control in Reducing Early Childhood Caries Among Young ChildrenStudy Protocol for A Cluster Randomized Controlled Trial. 2021. https://doi.org/10.21203/rs.3.rs$568884 / \mathrm{v} 1$.

17. Aliakbari E, Gray-Burrows KA, Vinall-Collier KA, Edwebi S, Marshman Z, McEachan RRC, et al. Homebased toothbrushing interventions for parents of young children to reduce dental caries: $\mathrm{A}$ systematic review. 2021; 31:37-79.

18. Collins LM, Kugler KC. Optimization of Behavioral, Biobehavioral, and Biomedical Interventions Advanced Topics. Cham: Springer International Publishing; 2018.

19. Kan MPH, Fabrigar LR. Theory of Planned Behavior. In: Encyclopedia of Personality and Individual Differences. Springer International Publishing; 2017. pp. 1-8.

20. Smith SR, Kroon J, Schwarzer R, Hamilton K. Parental social-cognitive correlates of preschoolers' oral hygiene behavior: A systematic review and meta-analysis. Soc Sci Med. 2020;264:113322.

21. Gao X, Lo ECM, Kot SCC, Chan KCW. Motivational Interviewing in Improving Oral Health: A Systematic Review of Randomized Controlled Trials. J Periodontol. 2014;85:426-37.

22. Anderson CN, Noar SM, Rogers BD. The Persuasive Power of Oral Health Promotion Messages: A Theory of Planned Behavior Approach to Dental Checkups Among Young Adults. Health Commun Health Commun. 2013;28:304-13.

23. Zhou N, Wong HM, McGrath C. Social story-based oral health promotion for preschool children with special healthcare needs: A 24-month randomized controlled trial. Community Dent Oral Epidemiol. 2020;48:415-22.

24. Motivational Interviewing Network of Trainers (MINT). Motivational Interviewing Training; 2013. https://motivationalinterviewing.org/(accessed $10 \mathrm{Jul}$ 2021). 
25. Fernández CE, Maturana CA, Coloma SI, Carrasco-Labra A, Giacaman RA. Teledentistry and mHealth for Promotion and Prevention of Oral Health: A Systematic Review and Meta-analysis. J Dent Res. 2021;26:220345211003828.

26. WhatsApp Inc. (Facebook, Inc.). WhatsApp [Internet]. 2021. Available from: https://whatsapp.com.

27. Jadhav HC, Dodamani AS, Karibasappa GN, Naik RG, Khairnar MR, Deshmukh MA, et al. Effect of Reinforcement of Oral Health Education Message through Short Messaging Service in Mobile Phones: A Quasi-Experimental Trial. 2016;2016:7293516.

28. Francis JJ, Johnston M, Robertson C, Glidewell L, Entwistle V, Eccles MP, et al. What is an adequate sample size? Operationalising data saturation for theory-based interview studies. Psychol Heal. 2010;25:1229-45.

29. Frankl SN, Shiere FR, Fogels HR. Should the parent remain with the child in the dental operatory? J Dent Child. 1962;29:150-63.

30. Sekhon M, Cartwright M, Francis JJ. Acceptability of healthcare interventions: An overview of reviews and development of a theoretical framework. BMC Health Serv Res BioMed Central Ltd. 2017;17:113.

31. Chilton NW, Fleiss JL. Design and analysis of plaque and gingivitis clinical trials. J Clin Periodontol. 1986;13:400-6.

32. Löe H. The Gingival Index, the Plaque Index and the Retention Index Systems. J Periodontol. 1967;38:610-6.

33. El Tantawi M, Bakhurji E, Al-Ansari A, Al-Khalifa KS, AlSubaie A. Influences of parents, close friends and classmates on four co-existing oral health practices in Saudi male teenagers. Acta Odontol Scand. 2017;75:137-43.

34. Effect Size in Statistics - The Ultimate Guide. Available from: https://www.spss-tutorials.com/effectsize/ (accessed July 10 2021).

35. Faul F, Erdfelder E, Lang AG, Buchner A. G*Power 3: A flexible statistical power analysis program for the social, behavioral, and biomedical sciences. Behav Res Methods. 2007;39(2):175-91.

36. Saghaei M. Random Allocation S. 2004. Available from:

https://mahmoodsaghaei.tripod.com/Softwares/randalloc.html (accessed 10 Jul 2021).

37. Collins LM, Trail JB, Kugler KC, Baker TB, Piper ME, Mermelstein RJ. Evaluating individual intervention components: making decisions based on the results of a factorial screening experiment. Transl Behav Med. 2014;4:238-51.

38. Corp IBM. Released 2020. IBM SPSS Statistics for Windows, Version 27.0. Armonk, NY: IBM Corp.

39. Carra MC, Detzen L, Kitzmann J, Woelber JP, Ramseier CA, Bouchard P. Promoting behavioural changes to improve oral hygiene in patients with periodontal diseases: A systematic review. J Clin Periodontol. 2020;47:72-89.

\section{Figures}




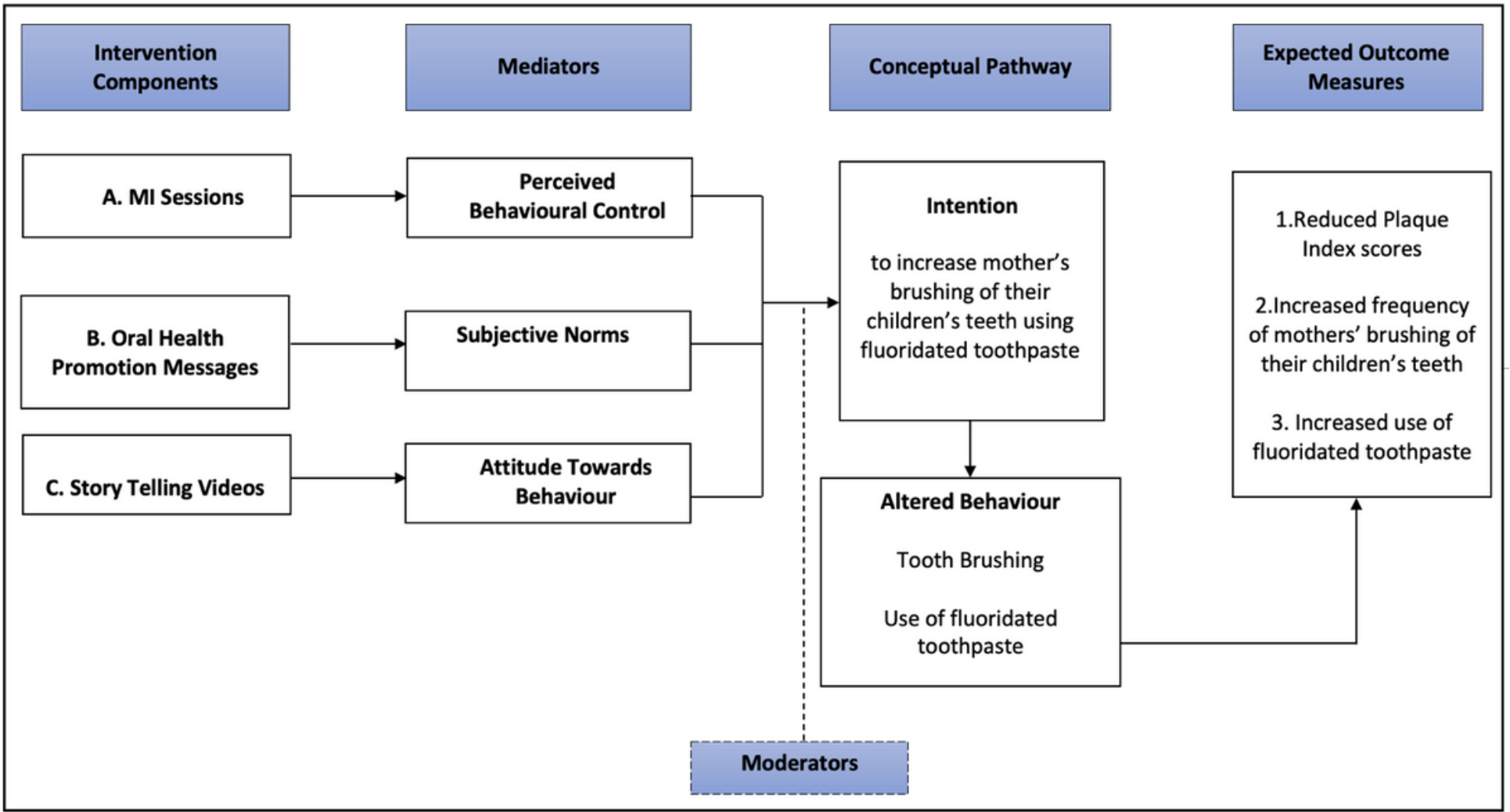

\section{Figure 1}

Conceptual Framework Based on the TPB 


\section{Screening for Eligibility}

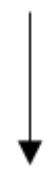

\section{Enrollment}

Written informed consent

Clinical examination of child

Baseline questionnaire administered to mothers

Randomization

\section{Interventions}

Constant Component

A.MI session (yes/no)

B. OHPM (yes/no)

C. Storytelling videos (yes/no)

\section{Figure 2}

Flow Chart of Participants Throughout the Factorial Trial of the Optimization Phase

\section{Supplementary Files}

This is a list of supplementary files associated with this preprint. Click to download. 
- Appendix.docx

- SPIRITChecklistDocument.doc 